\title{
Dynamical description of state mapping and discontinuous entanglement transfer for tripartite systems
}

\author{
M. Bina ${ }^{(a)}$, F. Casagrande, M. G. Genoni, A. Lulli and M. G. A. Paris \\ Dipartimento di Fisica dell'Università degli Studi di Milano - I-20133 Milano, Italy, EU and \\ CNISM, UdR Milano Università - I-20133 Milano, Italy, EU
}

received 14 December 2009; accepted in final form 2 May 2010

published online 1 June 2010

PACS 03.67.Mn - Entanglement measures, witnesses, and other characterizations PACS $42.50 . \mathrm{Pq}$ - Cavity quantum electrodynamics; micromasers

\begin{abstract}
We describe the transfer of quantum information and correlations from an entangled tripartite bosonic system to three localized qubits also in the presence of various dissipative effects. Optimal state mapping and entanglement transfer are shown in the framework of optical cavity quantum electrodynamics involving qubit-like radiation states and two-level atoms via the mediation of cavity modes. For an input GHZ state mixed with white noise we show the occurrence of sudden death and birth of tripartite entanglement, that is discontinuously exchanged among the subsystems. Each subsystem can exhibit, in different time intervals, tripartite entanglement of both GHZ and W class.
\end{abstract}

Copyright (c) EPLA, 2010

Entanglement is currently the key resource for quantum information (QI) processing [1], where it allows a number of achievements such as teleportation [2], cryptography [3] and enhanced measurements [4]. The deep meaning of multipartite entanglement, its quantification and detection [5], the possible applications, are the object of massive investigation. As a matter of fact, optical systems have been a privileged framework for encoding and manipulating quantum information, since bipartite and multipartite entanglement may be effectively generated either in the discrete or continuous variable (CV) regime. On the other hand, the development of QI also requires localized registers, e.g. for the storage of entanglement in quantum memories. Cavity quantum electrodynamics (CQED) [6] is a relevant scenario for this kind of investigations.

The general problem of transferring entanglement from bosonic systems to localized qubits for bipartite systems was recently addressed [7] also in the presence of some dissipative effects. In the framework of CQED the Hamiltonian description of entanglement exchange from radiation to two-level atoms was theoretically investigated in [8] and the effect of cavity mode decay was numerically analyzed in [9]. The literature also provides examples of similar investigations in other physical systems such as circuit QED [10] or collective spins of atomic ensembles [11]. In the case of tripartite systems the

(a) E-mail: matteo.bina@unimi.it problem of entanglement transfer was investigated in CQED for unitary dynamics [12,13]. In turn, tripartite entanglement of radiation in CV systems has been widely investigated both theoretically and experimentally [14-19] and, recently, photon number multipartite entanglement for qubit-like radiation states was demonstrated [20]. Another scheme for quantum state engineering has been proposed [21] allowing also entanglement purification [22].

In this letter we devote our attention to the full dynamical description of entanglement transfer from three entangled bosonic modes to three localized qubits through the action of a local environment. Our analysis provides a good framework for different physical systems and implementations. In particular, upon exploiting current advances in the optical regime of CQED [20,23,24], our scheme could be implemented with three entangled radiation modes, prepared in a qubit-like state, coupled by optical fibers to three separated optical cavities containing each one a trapped two-level atom. We first describe the whole system dynamics in the Hamiltonian case, showing the occurrence of optimal state mapping and entanglement transfer. In particular, an injected pure entangled state of radiation, carried by single-mode fibers, can be mapped onto the tripartite atomic subsystem, after a suitable interaction time among all nine parties. Switching off the external field at that time, the quantum correlations can be periodically mapped onto the tripartite atomic and cavity mode subsystems, according to a triple 
Jaynes-Cummings (JC) dynamics [25]. In the case of external radiation prepared in a mixed Werner state we suggest a way to observe the phenomenon of entanglement sudden death/birth (ESD/ESB) [26,27] for a tripartite system, though this is in general a still open problem related to the issue of multipartite entanglement measure. Furthermore we show that, during the time evolution, each subsystem (atoms or cavity modes) can alternatively exhibit different kind of entanglement, including genuine tripartite GHZ and W entanglement. Finally we take into account the main dissipative effects, such as the decay of cavity modes, atomic excitations and fiber modes, allowing the realistic investigation of multipartite entanglement transfer and swapping, shedding light on fundamental processes related to quantum interfaces and memories in quantum networks $[28,29]$.

We start describing the general scheme for an entangled three-mode bosonic system $(f)$, prepared in general in a mixed state, interacting with three qubits $(a)$ through their local environments $(c)$. In the interaction picture the system Hamiltonian has the form

$$
\begin{aligned}
\hat{\mathcal{H}}^{I}= & \hbar\left\{\sum_{J=A, B, C}\left[g_{J}\left(\hat{c}_{J} \hat{\sigma}_{J}^{\dagger}+\hat{c}_{J}^{\dagger} \hat{\sigma}_{J}\right)\right]\right. \\
& \left.+\sum_{J, K=A, B, C}\left[\nu_{J, K}(t)\left(\hat{c}_{J} \hat{f}_{K}^{\dagger}+\hat{c}_{J}^{\dagger} \hat{f}_{K}\right)\right]\right\} .
\end{aligned}
$$

The operators $\hat{c}_{J}, \hat{c}_{J}^{\dagger}\left(\hat{f}_{J}, \hat{f}_{J}^{\dagger}\right)$ are the annihilation and creation operators for the local environment (input bosonic) modes, while $\hat{\sigma}_{J}, \hat{\sigma}_{J}^{\dagger}$ are the lowering and raising operators for the target qubits in each subsystem $(J=A, B, C)$. We consider real coupling constants $g_{J}$ for the qubit-local environment interaction, whereas $\nu_{J, K}(t)$, that couple local environment and bosonic modes, are taken real and time dependent in order to simulate the interaction switching-off at a suitable time $t_{\text {off }}$.

The implementation of our scheme (sketched in fig. 1) may be realized in the optical regime of CQED by choosing a tripartite photon-number entangled field for the bosonic modes $(f)$, guided by optical fibers, and two-level atoms as the target qubits $(a)$. Each qubit is trapped in a onesided optical cavity, operating as the local environment (c). We consider the optical fibers in the short-fiber limit where radiation is carried by discrete modes [30]. So the entangled radiation, emerging, e.g., from cavities or beam splitters, can be coupled by each fiber mode to the final cavity mode. This is carried out by choosing $\nu_{J K}(t)=0$ if $J \neq K$. We also assume, for the sake of simplicity, $g_{J}=g_{A}$ and $\nu_{J, J}(t)=g_{A}$ with $0 \leqslant t \leqslant t_{o f f}$, for all $J$. The case of multi-mode coupling and different coupling constants will be considered elsewhere. We further assume optimal mode matching at the input cavity mirrors between incident and transmitted modes.

Now we add to system dynamics the main dissipative effects, i.e. the cavity losses at rate $\kappa_{c}$ due to the

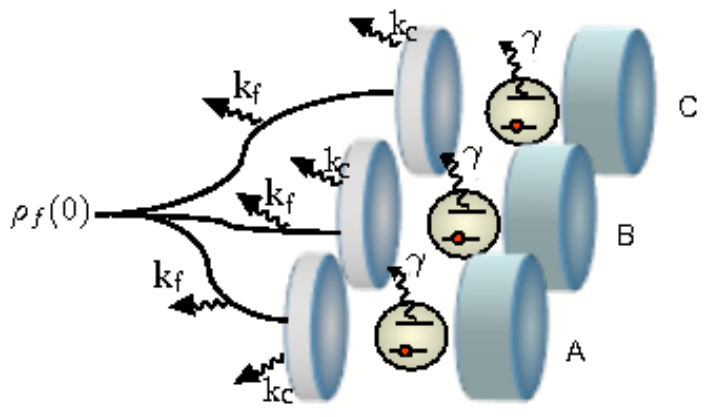

Fig. 1: (Colour on-line) Schematic diagram for entanglement transfer in a CQED framework. Three entangled radiation modes are coupled by optical fibers to three cavities each one containing an atomic qubit.

interaction with a thermal bath with a mean photon number $\bar{n}$, the atomic spontaneous emission with a decay rate $\gamma_{a}$, and a leakage of photons from the fibers at a rate $\kappa_{f}$. Hence the time evolution of the whole system is described by the following master equation (ME) in the Lindblad form for the density operator $\hat{\rho}(t)$ :

$$
\begin{aligned}
\dot{\hat{\rho}}= & -\frac{i}{\hbar}\left[\hat{\mathcal{H}}_{e}, \hat{\rho}\right]+\sum_{J=A, B, C}\left[\hat{C}_{f, J} \hat{\rho} \hat{C}_{f, J}^{\dagger}+\hat{C}_{c, J}^{(g)} \hat{\rho} \hat{C}_{c, J}^{(g) \dagger}\right. \\
& \left.+\hat{C}_{c, J}^{(l)} \hat{\rho} \hat{C}_{c, J}^{(l) \dagger}+\hat{C}_{a, J} \rho \hat{C}_{a, J}^{\dagger}\right],
\end{aligned}
$$

where the non-Hermitian effective Hamiltonian is

$$
\begin{aligned}
\hat{\mathcal{H}}_{e}= & \hat{\mathcal{H}}^{I}-\frac{i \hbar}{2} \sum_{J=A, B, C}\left[\hat{C}_{f, J}^{\dagger} \hat{C}_{f, J}+\hat{C}_{c, J}^{(g) \dagger} \hat{C}_{c, J}^{(g)}\right. \\
& \left.+\hat{C}_{c, J}^{(l) \dagger} \hat{C}_{c, J}^{(l)}+\hat{C}_{a, J}^{\dagger} \hat{C}_{a, J}\right] .
\end{aligned}
$$

The jump operators for the atoms are $\hat{C}_{a, J}=\sqrt{\gamma_{a}} \hat{\sigma}_{J}$, for the fibers $\hat{C}_{f, J}=\sqrt{\kappa_{f}} \hat{f}_{J}$, and for the cavity modes $\hat{C}_{c, J}^{(l)}=$ $\sqrt{\kappa_{c}(\bar{n}+1)} \hat{c}_{J}$ (loss of a photon) and $\hat{C}_{c, J}^{(g)}=\sqrt{\kappa_{c} \bar{n}} \hat{c}_{J}^{\dagger}$ (gain of a photon). We remark that in optical cavities thermal noise is negligible, spontaneous emission can be effectively suppressed, and single atoms can stay trapped even for several seconds [23]. From now on we consider dimensionless parameters, all scaled to the coupling constant $g_{A}$, and times $\tau=g_{A} t$, introducing the dimensionless switching-off time $\tau_{o f f}=g_{A} t_{o f f}$.

Starting from a qubit-like entangled state $\hat{\rho}_{f}(0)$ all subsystems $(a, c, f)$ behave as qubits and their entanglement can be described by combining the information from tripartite negativity [31], entanglement witnesses [32] for the two inequivalent classes GHZ and W [33], and recently proposed criteria for separability [34]. In fact, the tripartite negativity $E^{(\alpha)}(\tau)(\alpha=a, c, f)$, defined as the geometric mean of the three bipartite negativities [35], is an entanglement measure providing only a sufficient condition for entanglement detection, though its positivity guarantees GHZ-distillability.

We first illustrate the Hamiltonian dynamics $\left(\left\{\tilde{\kappa}_{f}, \tilde{\kappa}_{c}, \tilde{\gamma}_{a}\right\} \ll 1\right)$ for the external field prepared in 

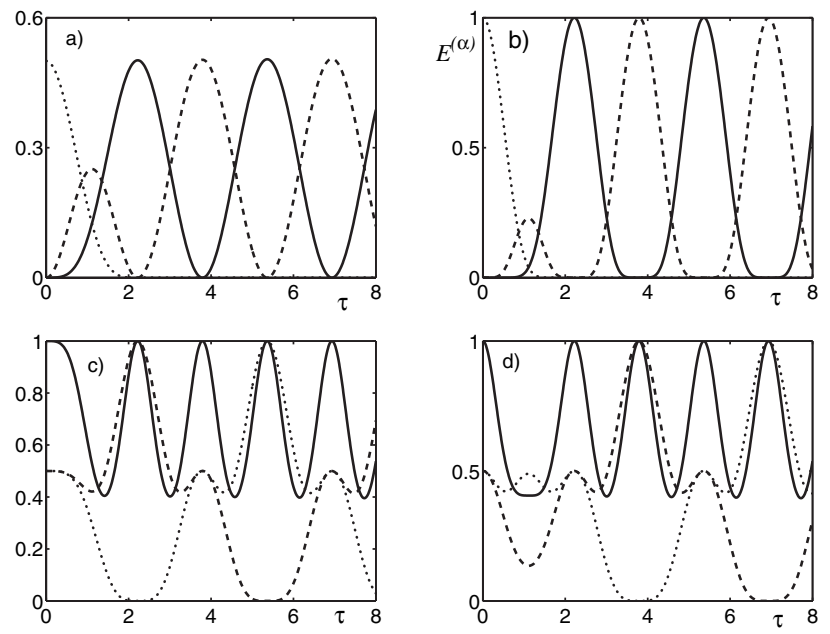

Fig. 2: Dynamics of the nine-qubit system for the external field initially prepared in a GHZ state. In (a) the average number of photons $N^{(c)}$ (dashed line), $N^{(f)}$ (dotted line) and the probability of excited state $p_{e}$ (solid line). In (b) the tripartite negativity $E^{(\alpha)}$ for atoms (solid line), cavity modes (dashed line) and external field (dotted line). In (c) the purity $\mu^{(a)}$ (solid line) and the fidelity $F_{\phi}^{(a)}$ with $\phi=0$ (dashed line), $\phi=\pi$ (dotted line). Finally, in (d) the purity $\mu^{(c)}$ (solid line) and the fidelity $F_{\phi}^{(c)}$ with $\phi=-\pi / 2$ (dashed line), $\phi=+\pi / 2$ (dotted line).

a qubit-like entangled pure state $|\Psi(0)\rangle_{f}$, atoms prepared in the lower state $|g g g\rangle_{a}$, and cavities in the vacuum state $|000\rangle_{c}$. Overall we are dealing with an interacting 9-qubit system where the input field is switched off at the time $\tau_{o f f}$, when it has completely fed the cavities and the atomic probability of excited state $p_{e}(\tau)$ reaches the maximum. In practice, the switch-off of the injected field can be obtained, e.g., by rotating the fiber polarization.

In fig. 2 we summarize the dynamics for the external field prepared in the GHZ state $|\Psi(0)\rangle_{f}=\left(|000\rangle_{f}+\right.$ $\left.|111\rangle_{f}\right) / \sqrt{2}$. We first describe the transient regime $0<$ $\tau \leqslant \tau_{o f f}$, where $\tau_{o f f}=\pi / \sqrt{2}$ : each input qubit transfers its excitation to the cavity which in turn passes it onto the atom (see fig. 2a). Each cavity mode, simultaneously coupled to the external field and to the atom, exchanges energy according to a Tavis-Cummings dynamics at an effective frequency $g_{A} \sqrt{2}[6,36]$ and the mean photon number $N^{(c)}(\tau) \equiv\left\langle\hat{c}^{\dagger} \hat{c}\right\rangle(\tau)$ in each cavity completes a cycle. In fig. $2 \mathrm{~b}$ we also see that the atomic tripartite negativity is always positive and $E^{(a)}\left(\tau_{o f f}\right)=1$, that is the value of the injected GHZ state. Until $\tau_{o f f}$ the dynamics maps the whole initial state $|\Psi(0)\rangle_{f} \otimes|000\rangle_{c} \otimes|g g g\rangle_{a}$ onto the pure state $|000\rangle_{f} \otimes|000\rangle_{c} \otimes|\Psi(0)\rangle_{a}$, where $|\Psi(0)\rangle_{a}$ is obtained from $|\Psi(0)\rangle_{f}$ by the correspondence $|0\rangle_{f} \rightarrow|g\rangle_{a}$ and $|1\rangle_{f} \rightarrow|e\rangle_{a}$. This is confirmed in fig. 2c by the time evolution of the purity $\mu^{(a)}(\tau)=\operatorname{Tr}_{a}\left[\hat{\rho}_{\alpha}^{2}(\tau)\right]$ and of the fidelity to the initial state $F^{(a)}(\tau)={ }_{a}\left\langle\Psi(0)\left|\hat{\rho}_{a}(\tau)\right| \Psi(0)\right\rangle_{a}$, where $\hat{\rho}_{a}(\tau)$ is the atomic reduced density operator. As for the cavity mode dynamics we note that (see figs. $2 \mathrm{~b}, \mathrm{~d}$ ) the local maximum of $E^{(c)}\left(\tau_{o f f} / 2\right)$ does not correspond

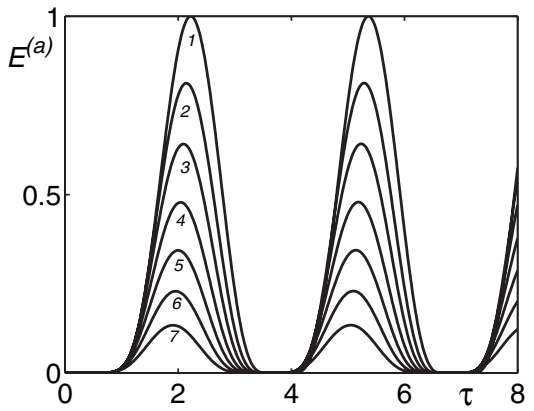

Fig. 3: Effect of cavity mirror transmittance $T$ on the dynamics of atomic entanglement $E^{(a)}: T=1.0$ (1), 0.9 (2), 0.8 (3), 0.7 (4), $0.6(5), 0.5(6), 0.4(7)$.

to a pure state, i.e. the initial state $|\Psi(0)\rangle_{f}$ cannot be exactly mapped onto the cavity modes during the transient regime. Therefore we have that entanglement is only partially transferred to the cavity modes but nevertheless this is enough for the building up of full atomic entanglement later on. This is quite different with respect to [13].

At the end of the transient regime the external radiation is turned off and the subsequent dynamics is described by a triple $\mathrm{JC}$ ruled by oscillations at the vacuum Rabi frequency $2 g_{A}$, hence with a dimensionless period $\pi$ as shown by cavity mean photon number and atomic probability in fig. 2a. The purities $\mu^{(a, c)}(\tau)$ in figs. 2c, d oscillate at a double frequency between pure entangled (maximum negativity) and separable (zero negativity) states. In particular, at times $\tau_{m}=\tau_{o f f}+m \pi$ $(m=0,1,2 \ldots)$ the atoms are in the entangled states $\hat{U}_{\phi}^{(a)}|\Psi(0)\rangle_{a}$, where $\hat{U}_{\phi}^{(a)}=\bigotimes_{J} e^{-i \phi \hat{\sigma}_{J}^{\dagger} \hat{\sigma}_{J}}$ is a local phase operator where $\phi=0 \quad(\phi=\pi)$ applies for even (odd) values of $m$, that are the peaks of $E^{(a)}(\tau)$ in fig. $2 \mathrm{~b}$. At times $\tau_{n}=\tau_{o f f}+\left(n+\frac{1}{2}\right) \pi(n=0,1,2 \ldots)$ the cavity mode states are obtained by applying $\hat{U}_{\phi}^{(c)}=\bigotimes_{J} e^{-i \phi \hat{c}_{J}^{\dagger} \hat{c}_{J}}$, where $\phi=-\frac{\pi}{2}\left(+\frac{\pi}{2}\right)$ for even (odd) values of $n$, to the state $|\Psi(0)\rangle_{c}$ derived from $|\Psi(0)\rangle_{f}$ by the correspondence $|0\rangle_{f} \leftrightarrow|0\rangle_{c}$ and $|1\rangle_{f} \leftrightarrow|1\rangle_{c}$. By choosing to turn off the external field at times shorter than $\tau_{o f f}$, we find a progressive degradation of the entanglement transfer to the atomic and cavity subsystems, as shown in fig. 3 . This effect is fully equivalent to the presence of a reduced cavity mirror transmittance. Notice that even for a $10 \%$ changes in the value of $\tau_{o f f}$, the fidelity $F^{(a)}\left(\tau_{o f f}\right)$ remains above $99.9 \%$, i.e. entanglement transfer is robust against fluctuations of the switching-off time and this feature is a relevant one in view of possible implementations. We also remark that state mapping is effective for any choice of $|\Psi(0)\rangle_{f}$ written in the generalized Schmidt decomposition of ref. [33], as well as for mixed states as described below.

Let us now consider the injected field excited in a Werner state $\hat{\rho}_{f}(0)=(1-p)|\mathrm{GHZ}\rangle\langle\mathrm{GHZ}|+\frac{p}{8} \hat{I}, \quad(0 \leqslant p \leqslant$ $1)$, for which it is possible to have full classification of entanglement: for $0 \leqslant p<\frac{2}{7}$ the state belongs to the GHZ 

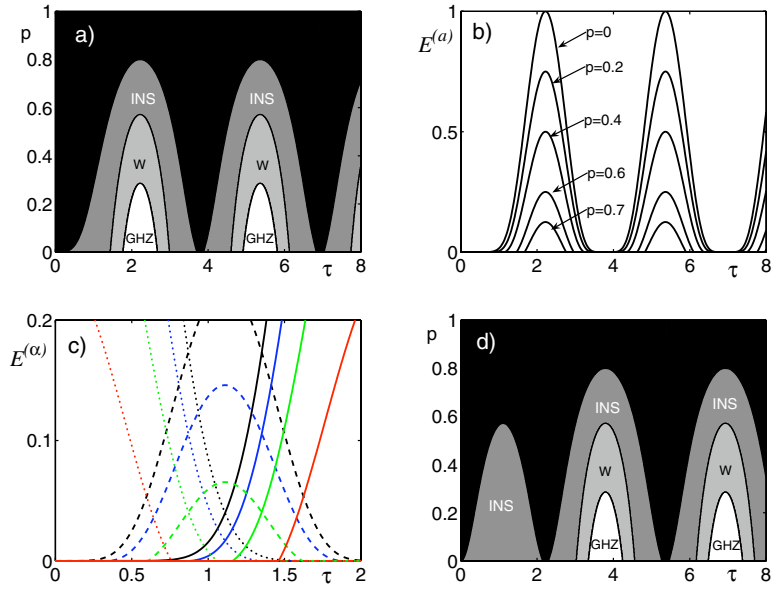

Fig. 4: (Colour on-line) ESD/ESB for external field in a GHZ state mixed with white noise. a) Regions in the plane $(\tau, p)$ for atomic entanglement of type GHZ, W, INS, and fully separable (black). b) Sections $E^{(a)}(\tau)$ for selected values of $p$. c) Zoom on $E^{(\alpha)}\left(\tau_{o f f} / 2\right)$ for field (dotted line), cavity modes (dashed line), and atoms (solid line) with $p=0$ (black), $p=0.2$ (blue), $p=0.4$ (green), $p=0.6$ (red). d) Classification for cavity mode entanglement.

class and to the $\mathrm{W}$ class up to $p=\frac{4}{7}$. The tripartite negativity is positive up to $p=4 / 5$ whereas the state is clearly inseparable under all bipartitions (from now on we denote this class by INS), i.e. it cannot be written as convex combination of biseparable states and finally, for $4 / 5 \leqslant$ $p \leqslant 1$ it is known that the state is fully separable $[34,37]$.

The system dynamics can be divided again into a transient and an oscillatory regime, and the state mapping of $\hat{\rho}_{f}(0)$ onto atoms (cavity modes) still occurs at certain finite times $\tau_{m}\left(\tau_{n}\right)$. Out of these times the density matrices of all subsystems lose the form of a GHZ state mixed with white noise but still preserve invariance under all permutations of the three qubits and present only one non-vanishing coherence as in $\hat{\rho}_{f}(0)$. This greatly helps us in the entanglement classifications in the plane $(\tau, p)$ as shown in fig. 4. In fact, in the regions where $E^{(\alpha)}(\tau)>0$ but out of the $\mathrm{W}$ class we can exclude the biseparability. The full separability criteria in [34] are violated only where $E^{(a)}(\tau)>0$ so that if $E^{(a)}(\tau)=0$ the state may be fully separable or biseparable. Nevertheless, in the latter case the state should be symmetric and biseparable under all bipartitions and hence it is fully separable [38]. For any fixed value of $p$ in the range $0<p<4 / 5$ we thus show the occurrence of entanglement sudden death and birth at the boundaries between fully separable and inseparable states. In particular, for $0<p<4 / 7$ we find genuine tripartite ESD and ESB phenomena. Furthermore note that, for a fixed value of $p$, the atomic state may exhibit transition from W to GHZ entanglement class and viceversa. The same effect is shown by the cavity modes after the transient (see fig. 4d). The W-GHZ transition is allowed by the non-unitarity of the partial trace over non-atomic degrees of freedom, which implies that the overall map on the initial three qubits is not SLOCC. We also notice that for times $\tau \geqslant \tau_{\text {off } f}$ we can solve exactly the triple JC dynamics, thus confirming our numerical results and providing generalization of the results of ref. [39] to mixed states.

In fig. $4 \mathrm{~b}$ we see that, for increasing values of $p$, there is an increase of both the slope of $E^{(a)}(\tau)$ and the time interval of full separability. In fig. $4 \mathrm{c}$ we show in detail the transient dynamics of the tripartite negativities $E^{(\alpha)}(\tau, p)(\alpha=a, c, f)$ in the crucial region around $\tau_{o f f} / 2$. We consider some values of $p$ where the atoms exhibit in times different classes of entanglement. We see that for $p=$ 0.2 , where the input state has GHZ class entanglement, the ESB of subsystems $(c),(a)$ anticipates the ESD of $(f),(c)$, and there is an interval around $\tau_{o f f} / 2$ where all three subsystems are entangled (of INS-type). As $p$ grows, hence the initial state becomes more noisy, the effects of ESD occur earlier and those of ESB later. For $p=0.4$, involving W-class entanglement, only at most two subsystems are simultaneously entangled (first $(f),(c)$ and then $(c),(a))$. For $p=0.6$, involving only entanglement of INS-type, the cavity modes do not entangle at all (see fig. 4d). They physically mediate the discontinuous entanglement transfer from $(f)$ to $(a)$, where for $p \rightarrow 4 / 5$ the time interval without any entanglement increases while the entanglement level vanishes.

In the perspective of an experimental implementation for QI purposes an important issue is the detrimental effect of dissipation on both state mapping and entanglement transfer. Here we provide some examples, obtained by solving the full ME through Monte Carlo Wave Function method [40], in order to give a quantitative behavior of the systems in realistic conditions. Work along this line is in progress and more detailed results will be reported elsewhere. Let us start with external field prepared in a GHZ pure state and analyze the effect of cavity decay rates in the range $0<\tilde{\kappa}_{c} \leqslant 0.5$ for negligible values of all other decay rates. We consider the fidelities $F^{(\alpha)}\left(\tau_{m, n}\right)$ and the tripartite negativities $E^{(\alpha)}\left(\tau_{m, n}\right)(\alpha=c, f)$ at the first peaks $(m=n=0)$ as function of $\tilde{\kappa}_{c}$. In the left panel of fig. 5 we see that all these quantities can be well fitted by exponential functions, with decay rates $\beta_{F}^{(a)}=0.75$, $\beta_{E}^{(a)}=1.09$ for the atomic subsystem, and $\beta_{F}^{(c)}=1.80$, $\beta_{E}^{(c)}=2.94$ for the cavity modes. As one may expect, quantum state mapping and entanglement transfer are by far more efficient onto atomic than cavity qubits. For instance, if $\tilde{\kappa}_{c}=0.1$ we obtain a state mapping onto the atoms (cavity modes) with a fidelity of $\cong 0.93(\cong 0.83)$. Upon adding a non-zero atomic decay we see that this is a minor effect compared to cavity decay. As for example, for an atomic decay rate $\tilde{\gamma}_{a}=0.03$ and in the presence of cavity decay with a rate $\tilde{\kappa}_{c}=0.1$, the fidelity of the atomic (cavity mode) subsystem reduces by $4.4 \%$ (8.9\%). Finally, we address the effect of fiber losses, which is of course relevant only up to the time $\tau_{\text {off }}$. We evaluated the effects of decay rates $\tilde{\kappa}_{f}$ up to 1.0 for negligible values 

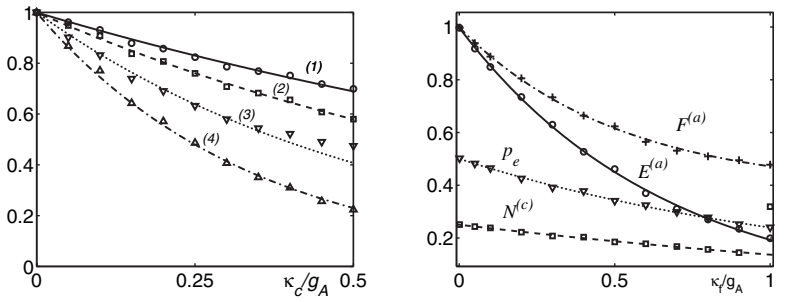

Fig. 5: Left: effect of cavity dissipation. Tripartite negativities and fidelities vs. $\tilde{\kappa}_{c} \equiv \kappa_{c} / g_{A}: F^{(a)}\left(\tau_{0}\right)(1), E^{(a)}\left(\tau_{0}\right)(2), F^{(c)}\left(\tau_{0}\right)$ (3), $E^{(c)}\left(\tau_{0}\right)(4)$. Right: effect of fiber dissipation. Atomic tripartite negativity $E^{a}$ (solid line), fidelity $F^{a}$ (dash-dotted line), and atomic probability $p_{e}$ (dotted line) at time $\tau_{o f f}$; cavity mean photon number $N^{(c)}$ (dashed line) at time $\tau_{o f f} / 2$, vs. $\tilde{\kappa}_{f} \equiv \kappa_{f} / g_{A}$ for $\tilde{\kappa}_{c} \ll 1, \tilde{\gamma}_{a} \ll 1$.

of atomic and cavity decay rates $\left(\tilde{\kappa}_{c} \ll 1, \tilde{\gamma}_{a} \ll 1\right)$ (see the right panel of fig. 5). We show the effect of fiber losses $\tilde{\kappa}_{f}$ on cavity field mean photon number $N^{(c)}\left(\tau_{o f f} / 2\right)$ and atomic excitation probability $p_{e}\left(\tau_{o f f}\right)$ and we see that the amount of energy transferred to the atoms and to the cavity modes decreases exponentially for increasing values of $\tilde{\kappa}_{f}$; the decay rates are $\cong 0.42$ and $\cong 0.82$, respectively. The behavior of the tripartite negativity $E^{(a)}\left(\tau_{0}\right)$ and the fidelity $F^{(a)}\left(\tau_{0}\right)$ at the first peak can be described by exponential functions of $\tilde{\kappa}_{f}$, whose decay rates are $\cong 1.51$ and $\cong 1.95$, respectively.

In this letter we have addressed the transfer of quantum information and entanglement from three qubit-like radiation modes to three localized qubits mainly in a CQED setting. We have provided the full description of the dynamics including the finite time duration of the interaction. In the case of a GHZ input state mixed with white noise, we achieve the full characterization of the separability properties of the tripartite subsystems, showing the occurrence of entanglement sudden death effects at the tripartite level, deriving the conditions for (repeated) discontinuous exchange of quantum correlations among the subsystems. This is an issue of fundamental interest as well as worth investigating for all applications in quantum information processing, in particular computing and error correction, where disentanglement, which may be faster than decoherence, has to be carefully controlled. Furthermore we have shown that the atoms/cavities subsystems can alternatively exhibit in time genuine tripartite entanglement of both GHZ and W type.

$$
* * *
$$

This work has been partially supported by the CNRCNISM convention.

\section{REFERENCES}

[1] Nielsen M. A. and Chuang I. L., Quantum Computation and Quantum Information (Cambridge University Press) 2000.

[2] Bennett C. H. et al., Phys. Rev. Lett., 70 (1993) 1895.
[3] Gisin N. et al., Rev. Mod. Phys., 74 (2002) 1458.

[4] D'Ariano G. M., Lo Presti P. and Paris M. G. A., Phys. Rev. Lett., 87 (2001) 270404.

[5] Gühne O. and Toth G., Phys. Rep., 474 (2009) 1.

[6] Haroche S. and Raimond J. M., Exploring the Quantum (Oxford University Press) 2006.

[7] Paternostro M., Son W. and Kim M. S., Phys. Rev.Lett., 92 (2004) 197901.

[8] Son W. et al., J. Mod. Opt., 49 (2002) 1739; PAternostro M. et al., Phys. Rev. A, 70 (2004) 022320; Zou J. et al., Phys. Rev. A, 73 (2006) 042319; Casagrande F., Lulli A. and Paris M. G. A., Phys. Rev. A, 75 (2007) 032336; Paternostro M., Adesso G. and Campbell S., Phys. Rev. A, 80 (2009) 062318.

[9] Casagrande F., Lulli A. and Paris M. G. A., Eur. Phys. J. ST, 160 (2008) 71.

[10] Paternostro M. et al., Phys. Rev. B, 69 (2004) 214502.

[11] Hald J. et al., J. Mod. Opt., 47 (2000) 2599; JulsGAARD B. et al., Nature, 432 (2004) 482.

[12] Lee J. et al., Phys. Rev. Lett., 96 (2006) 080501.

[13] Casagrande F., Lulli A. and Paris M. G. A., Phys. Rev. A, 79 (2009) 022307.

[14] Zhang J. et al., Phys. Rev. A, 66 (2002) 032318; Jing J. et al., Phys. Rev. Lett., 90 (2003) 167903; Aoki T. et al., Phys. Rev. Lett., 91 (2003) 080404.

[15] Bondani M. et al., Opt. Lett., 29 (2004) 180; Ferraro A. et al., J. Opt. Soc. Am. B, 21 (2004) 1241; Allevi A. et al., Las. Phys., 16 (2006) 1451.

[16] Allevi A. et al., Phys. Rev. A, 78 (2008) 063801.

[17] Furusawa A. et al., Science, 282 (1998) 706.

[18] Bradley A. S. et al., Phys. Rev. A, 72 (2005) 053805; Pfister O. et al., Phys. Rev. A, 70 (2004) 020302(R).

[19] Villar A. S. et al., Phys. Rev. Lett., 97 (2006) 140504.

[20] PAPP S. B. et al., Science, 324 (2009) 764.

[21] Nielsen A. E. B. et al., Phys. Rev. A, 81 (2010) 043832.

[22] Lougovski P., Solano E. and Walther H., Phys. Rev. A, 71 (2005) 013811.

[23] Nussmann S. et al., Nat. Phys., 1 (2005) 122; Fortier K. M. et al., Phys. Rev. Lett., 98 (2007) 233601; Ye J., Kimble H. J. and Satori H., Science, 320 (2008) 1734.

[24] Mundt A. B. et al., Phys. Rev. Lett., 89 (2002) 103001; Keller M. et al., Nature, 431 (2004) 1075.

[25] Jaynes E. T. and Cummings F. W., Proc. IEEE, 51 (1963) 89.

[26] Zyczkovski K. et al., Phys. Rev. A, 65 (2001) 012101; Simon C. and Kempe J., Phys. Rev. A, 65 (2002) 052327; Dodd P. J. and Halliwell J. J., Phys. Rev. A, 69 (2004) 052105; Yu T. and Eberly J. H., Phys. Rev. Lett., 93 (2004) 140404; López C. E. et al., Phys. Rev. Lett., 101 (2008) 080503.

[27] Almeida M. P. et al., Science, 316 (2007) 579; Laurat J. et al., Phys. Rev. Lett., 99 (2007) 180504; SAlles A. et al., Phys. Rev. A, 78 (2008) 022322.

[28] Cirac J. I. et al., Phys. Rev. Lett., 78 (1996) 3221; Boozer A. D. et al., Phys. Rev. Lett., 98 (2007) 193601.

[29] Serafini A. et al., Phys. Rev. A, 73 (2006) 022312.

[30] Pellizzari T., Phys. Rev. Lett., 79 (1997) 5242; van Enk S. J. et al., Phys. Rev. A, 59 (1999) 2659; Serafini A., Mancini S. and Bose S., Phys. Rev. Lett., 96 (2006) 010503. 
[31] Sabin C. and Garcia-Alcaine G., Eur. Phys. J. D, 48 (2008) 435.

[32] Acin A. et al., Phys. Rev. Lett., 87 (2001) 040401.

[33] Dür W., Vidal G. and Cirac J. I., Phys. Rev. A, 62 (2000) 062314.

[34] Gühne O. and Seevinck M., New J. Phys., 12 (2010) 053002 .

[35] Vidal G. and Werner R. F., Phys. Rev. A, 65 (2002) 032314 .
[36] Tavis M. and Cummings F. W., Phys. Rev., 188 (1969) 692.

[37] Pittenger A. O. and Rubin M. H., Opt. Commun., 179 (2000) 447.

[38] Kraus B., PhD Thesis available on line as MPQ282.

[39] Ge M., Zhu L.-F. and Qiu L., Commun. Theor. Phys., 49 (2008) 1443.

[40] Dalibard J., Castin Y. and Mølmer K., Phys. Rev. Lett., 68 (1992) 580. 